\title{
The High Resolution Telescope (HRT) of the Polarimetric and Helioseismic Imager (PHI) onboard Solar Orbiter
}

Gandorfer, A., Grauf, B., Staub, J., Bischoff, J., Woch, J., et al.

A. Gandorfer, B. Grauf, J. Staub, J. Bischoff, J. Woch, J. Hirzberger, S. K. Solanki, A. Álvarez-Herrero, P. García Parejo, W. Schmidt, R. Volkmer, T. Appourchaux, J. C. del Toro Iniesta, "The High Resolution Telescope (HRT) of the Polarimetric and Helioseismic Imager (PHI) onboard Solar Orbiter," Proc. SPIE 10698, Space Telescopes and Instrumentation 2018: Optical, Infrared, and Millimeter Wave, 106984N (6 July 2018); doi: 10.1117/12.2311816 United States 


\title{
The High Resolution Telescope (HRT) of the Polarimetric and Helioseismic Imager (PHI) onboard Solar Orbiter
}

\author{
A. Gandorfer ${ }^{\mathrm{a}}$, B. Grauf ${ }^{\mathrm{a}}$, J. Staub ${ }^{\mathrm{a}}$, J. Bischoff ${ }^{\mathrm{a}}$, J. Woch ${ }^{\mathrm{a}}$, J. Hirzberger ${ }^{\mathrm{a}}$, S.K. Solanki ${ }^{\mathrm{a}}$, \\ A. Álvarez-Herrero ${ }^{\mathrm{b}}$, P. García Parejo ${ }^{\mathrm{b}}$, W. Schmidt ${ }^{\mathrm{c}}$, R. Volkmer ${ }^{\mathrm{c}}$, T. Appourchaux ${ }^{\mathrm{d}}$, and \\ J.C. del Toro Iniesta ${ }^{\mathrm{e}}$ \\ ${ }^{a}$ Max Planck Institute for Solar System Research, Justus-von-Liebig-Weg 3, Göttingen, \\ Germany \\ ${ }^{\mathrm{b}}$ Instituto Nacional de Técnica Aeroespacial, Torrejon de Ardoz, Spain \\ 'Kiepenheuer-Institut für Sonnenforschung, Schöneckstrasse 6, Freiburg, Germany \\ dinstitut d'Astrophysique Spatiale, Centre universitaire d'Orsay, 91405 Orsay, France \\ eInstituto de Astrofísica de Andalucía (IAA - CSIC), Apartado 3004, Granada, Spain
}

\begin{abstract}
Solar Orbiter is a joint mission of ESA and NASA scheduled for launch in 2020. Solar Orbiter is a complete and unique heliophysics mission, combining remote sensing and in-situ analysis; its special elliptical orbit allows viewing the Sun from a distance of only $0.28 \mathrm{AU}$, and - leaving the ecliptic plane - to observe the solar poles from a hitherto unexplored vantage point. One of the key instruments for Solar Orbiter's science is the "Polarimetric and Helioseismic Imager" (PHI), which will provide maps of the solar surface magnetic fields and the gas flows on the visible solar surface. Two telescopes, a full disc imager, and a high resolution channel feed a common Fabry-Perot based tunable filter and thus allow sampling a single Fraunhofer line at $617.3 \mathrm{~nm}$ with high spectral resolution; a polarization modulation system makes the system sensitive to the full state of polarization. From the analysis of the Doppler shift and the magnetically induced Zeeman polarization in this line, the magnetic field and the line-of-sight gas motions can be detected for each point in the image. In this paper we describe the opto-mechanical system design of the high resolution telescope. It is based on a decentred Ritchey-Chrétien two-mirror telescope. The telescope includes a Barlow type magnifier lens group, which is used as in-orbit focus compensator, and a beam splitter, which sends a small fraction of the collected light onto a fast camera, which provides the error signals for the actively controlled secondary mirror compensating for spacecraft jitter and other disturbances. The elliptical orbit of the spacecraft poses high demands on the thermo-mechanical stability. The varying size of the solar disk image requires a special false-light suppression architecture, which is briefly described. In combination with a heat-rejecting entrance window, the optical energy impinging on the polarimetric and spectral analysis system is efficiently reduced. We show how the design can preserve the diffraction-limited imaging performance over the design temperature range of $-20^{\circ} \mathrm{C}$ to $+60^{\circ} \mathrm{C}$. The decentred hyperbolical mirrors require special measures for the inter-alignment and their alignment with respect to the mechanical structure. A system of alignment flats and mechanical references is used for this purpose. We will describe the steps of the alignment procedure, and the dedicated optical ground support equipment, which are needed to reach the diffraction limited performance of the telescope. We will also report on the verification of the telescope performance, both - in ambient condition - and in vacuum at different temperatures.
\end{abstract}

\section{INTRODUCTION - THE PHI INSTRUMENT}

The Polarimetric and Helioseismic Imager PHI (Gandorfer et al., 2011; Solanki et al., 2014) is one out of ten scientific instruments onboard the ESA/NASA Solar Orbiter Mission (Marsden et al., 2011; Müller et al., 2013). PHI is a diffraction limited, wavelength tunable, quasi-monochromatic, polarisation-sensitive imager. These

Further author information: Send correspondence to Achim Gandorfer

gandorfer@mps.mpg.de, +49 551384 979-397

Space Telescopes and Instrumentation 2018: Optical, Infrared, and Millimeter Wave, edited by Makenzie Lystrup,

Howard A. MacEwen, Giovanni G. Fazio, Proc. of SPIE Vol. 10698, 106984N

(C) 2018 SPIE · CCC code: $0277-786 X / 18 / \$ 18 \cdot$ doi: $10.1117 / 12.2311816$

Proc. of SPIE Vol. 10698 106984N-1

Downloaded From: https://www.spiedigitallibrary.org/conference-proceedings-of-spie on 07 Jul 2020 
capabilities are needed to infer the magnetic field (via spectropolarimetry of a Zeeman-split spectral line) and the line-of-sight (LOS) velocity (via spectroscopy of the Doppler shift of the spectral line) of the region targeted by the spacecraft (S/C). PHI consists of two telescopes: The High Resolution Telescope (HRT) provides a restricted field-of-view (FOV) of 16.67 arcmin squared and achieves a spatial resolution that, near the closest perihelion pass, will correspond to about $200 \mathrm{~km}$ on the Sun. The Full Disk Telescope (FDT), with a FOV of $2.1^{\circ}$ diameter and a pixel size of $717 \mathrm{~km}$ (at $0.28 \mathrm{AU}$ ), provides a complete view of the full solar disk during all orbital phases. The two telescopes are used sequentially, and their selection is made by a Feed Selection Mechanism (FSM), a turnable fold mirror, which passes the light from the HRT, or the FDT, on towards the common optical path, while blocking the light of the other channel, respectively. An internal Image Stabilization System (ISS) is necessary to guarantee the required image stability in the HRT channel. A Correlation Tracker (CT) provides the necessary correction signals to an internal tip-tilt mirror (Volkmer et al., 2012). In order to limit the amount of light entering the instrument, two Heat Rejecting Entrance Windows (HREWs), one for each telescope, are mounted on the S/C heat-shield.

For the spectral analysis, PHI uses a Fabry-Perot solid state etalon to isolate a band-pass of the order of $10 \mathrm{pm}$. The etalon is made from lithium niobate $\left(\mathrm{LiNbO}_{3}\right)$ and is located inside a temperature stabilized oven. The etalon is used in the so-called telecentric mount (it resides close to an internal image plane, and the pupil is at infinity) in order to equalize the illumination geometry across the field-of-view. $\mathrm{LiNbO}_{3}$ etalons have been commercially available and have been developed and qualified in the past for solar magnetographs (in particular for the IMaX instrument, Martínez-Pillet et al. (2011) onboard the stratospheric balloon-borne observatory Sunrise (Barthol et al., 2011; Solanki at al. (2010). Gensemer and Farrant (2014) report on the fabrication and the metrology of the special etalon developed for PHI. The etalon based filtergraph (FG) extracts a spectral portion of the light inside the selected science spectral line (Fe I $617.3 \mathrm{~nm}$ ) and at a nearby continuum point. The FG provides a tuning range of $\pm 0.06 \mathrm{~nm}$, which is required for compensating the $\mathrm{S} / \mathrm{C}$ radial velocity of $\pm 23 \mathrm{~km} / \mathrm{s}$ plus the range required to scan the spectral line (50 pm, depending on the observing mode). Finally, the monochromatic image is demagnified by the camera lens system on the $2048 \times 2048$ pixel APS detector of the Focal Plane Assembly (FPA). Polarization analysis is based on sequential polarization modulation and demodulation of the resulting intensity differences (differential photometry). Each telescope uses its own Polarisation Modulation Package (PMP) including two nematic liquid crystal variable retarders and a linear polarizer as the analyzer. The camera therefore sees an intensity, which is modulated in a characteristic way, as a function of the original polarization.

\section{HRT OPTICAL DESIGN}

The full light path of the HRT with the relevant components is depicted in Fig. 1. In this picture the HREW is not shown, since it resides in the heat shield of the S/C.

\subsection{Common Optical Path}

The common optical path of PHI contains the filtergraph (FG) with the Fabry-Perot etalon and the reimaging optics from the filtergraph to the science focal plane array (FPA). PHI achieves monochromatic imaging by placing the etalon in an internal focal plane; for equal illumination of the etalon over the entire FOV, the pupil is imaged to infinity (telecentric configuration). Pupil imaging is done by two lenses, which form the entrance and the exit of the FG compartment, respectively, and serve as thermal IR shields for better insulation of the temperature sensitive etalon. Inside the FG two narrow-band prefilters are placed around the etalon, which serve as order sorters.

Figure 2 shows a detailed view of the FG optics components (coloured in red). OVEN_L1 picks up the intermediate exit pupil of the HRT and the FDT telescopes, respectively, and images the pupil to infinity. Prefilter 1 (PF1) is a narrow $(0.28 \mathrm{~nm})$ band-pass filter which blocks the side transmission maxima of the etalon. Prefilter 2 (PF2) is a broad band blocking filter suppressing like PF1 out of science band wavelengths. The OVENL_L2 lens reimages the pupil before the camera optics. In the pupil image a slightly oversized aperture is located (Lyot type stop), which blocks ghost images generated from the etalon and the two prefilters. Both prefilters are wedged by $1^{\circ}$, thus avoiding ghosts and interference fringes in the science focus. The distance between the etalon focus and the two adjacent components PF1 and the etalon is $15 \mathrm{~mm}$. This distance was considered sufficiently out of focus in order to prevent dust particles from being imaged onto the science detector. The depth of focus within the oven is approximately $4 \mathrm{~mm}$ for both telescopes (HRT and FDT). 


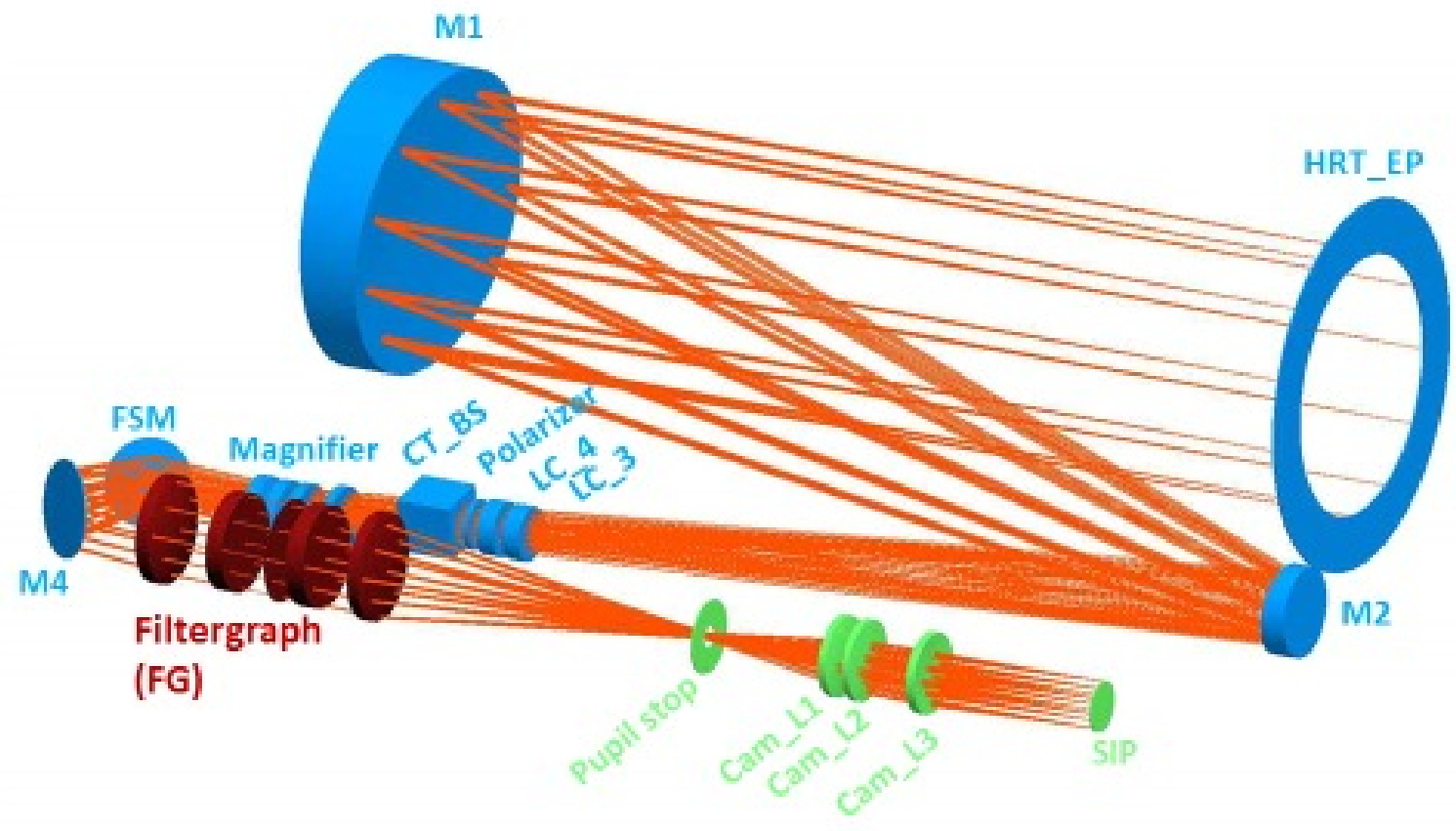

Figure 1: Optical design of the complete HRT path up to the science focal plane (SIP).

\subsection{HRT telescope}

The HRT-telescope is an off-axis Ritchey-Chrétien type. The off-axis configuration has been selected for three reasons: 1 . The design without central obstruction provides a better contrast transmission at intermediate spatial frequencies. 2. It avoids that the mounting of the secondary mirror is exposed to direct sunlight, which would aggravate the thermal situation in the telescope. 3. The decentred design offers the possibility for very efficient suppression of ghost images in the optical path after the telescope. A decentred optical system must be regarded as an asymmetric part of a larger, symmetric "parent" system. All optical components up to the science focal plane are symmetrically placed (i.e. normal) with respect to the optical axis of the parent telescope. Due to the decentred aperture the light rays are all oblique to the components, and thus the ghosts are reflected out of the system and do not coincide with their original beams. The only optical component that must be used with normal incidence to the oblique beam is the etalon, which for this reason is inclined with respect to the optical axis of the system. Since the image plane is normal to the optical axis, this implies that there are no ghosts, which can beat between the etalon and the detector. Further information about ghost/false-light suppression will be given below.

The filtergraph optics must be fed with an F-number of at least 55, so that the effective focal length at the etalon focus corresponds to $7.7 \mathrm{~m}$. This cannot be provided by a single two-mirror telescope within the given volume. Therefore the focal length of the telescope was selected as long as possible in order to fit the telescope in the dedicated space and still having reasonable tolerances. The secondary magnification of the telescope is 3.34 and the F-number of the (rotationally symmetric) parent telescope corresponds to 5.2. For further magnification a lens group consisting of four lenses (magL1 to magL4) is used. The focus of the two mirror system lies virtually behind the magnifier, therefore the lens group acts as a Barlow lens. The virtual image is picked up by the magnifier group and projected in the centre of the FG. 


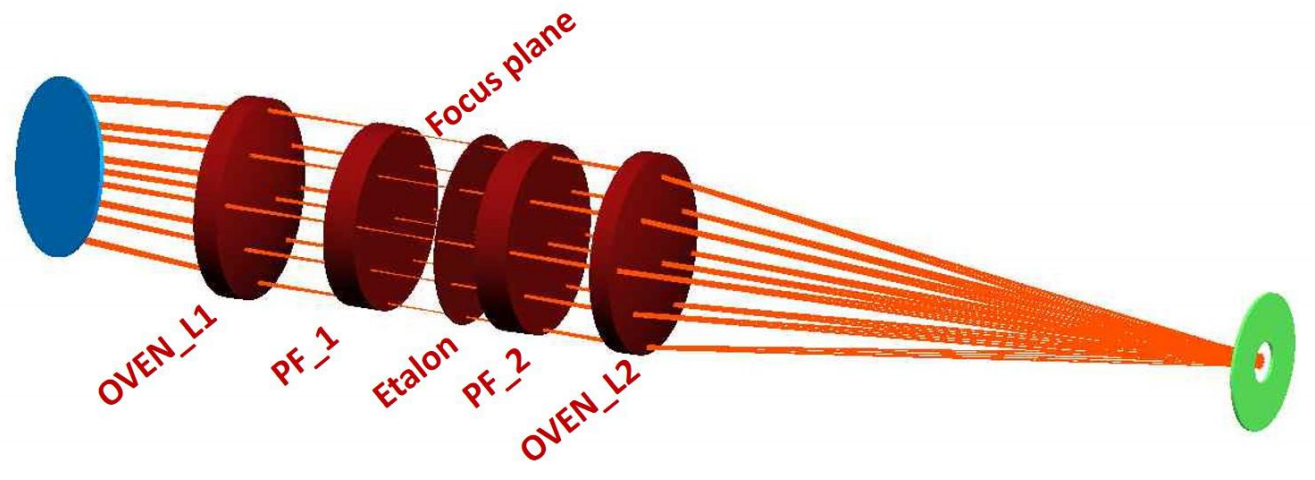

Figure 2: Optical design of the filtergraph (FG) path. The etalon is located close to an intermediate image plane with the pupil at infinity. The telecentric arrangement ensures equal illumination of the etalon over the full FOV. Two oven lenses OVEN_L1 and OVEN_L2 cover the closed compartment, which is used for temperature control of the etalon. The prefilters for order selection are wedged and inclined, such that there are no parallel surfaces inside the filtergraph (with the exception of the etalon itself). OVEN_L2 forms a real image of the pupil, where a Lyot stop is placed.

\subsubsection{Further functionalities}

The magnifying lens group can be shifted by a mechanism and acts as a defocus compensator during flight. For polarisation modulation the liquid crystals LC3 and LC4 are used. A linear polarizer acts as an analyzer. For details on the polarisation modulators we refer to Álvarez-Herrero et al. (2015). The beam-splitter cube sends a fraction of the incoming HRT light continuously to the correlation tracker camera (Carmona et al., 2014).

\section{HRT THERMO-OPTO-MECHANICAL DESIGN}

\subsection{Structure design}

Main driver for the PHI mechanical concept are the tight positional tolerances between the primary and secondary mirror of the HRT telescope (see Table 1), which have to be ensured over the full operational temperature range $\left(-20^{\circ} \mathrm{C}\right.$ to $\left.+60^{\circ} \mathrm{C}\right)$.

\begin{tabular}{ll}
\hline Parameter & Value \\
\hline Focus & $0.1 \mathrm{~mm}$ \\
Decenter & $0.04 \mathrm{~mm}$ \\
Rotation & $0.05^{\circ}$ \\
Tilt & $0.005^{\circ}$ \\
\hline
\end{tabular}

Table 1: Positional Tolerances of M1/M2

The values reported in Table 1 are one order of magnitude more critical than for each other optical component in the optical path of the HRT channel, and therefore drive the mechanical design of PHI.

The next two boundary conditions constraining the selection of a suitable mechanical concept are the need to accommodate 13 subsystems and to ensure a global stiffness of the unit corresponding to a first eigenmode above $140 \mathrm{~Hz}$. These demanding requirements lead to the selection of a more classical telescope structure with six struts (made from carbon fibre reinforced plastic and tailored to zero thermal expansion) and two end blocks, rather than a standard optical bench design.

As said, the optical performance of the HRT depends on tight mechanical tolerances for the relative positioning of the two mirrors M1 and M2. Especially the lateral decenter tolerances of M2 wrt. M1 are critical. For the 

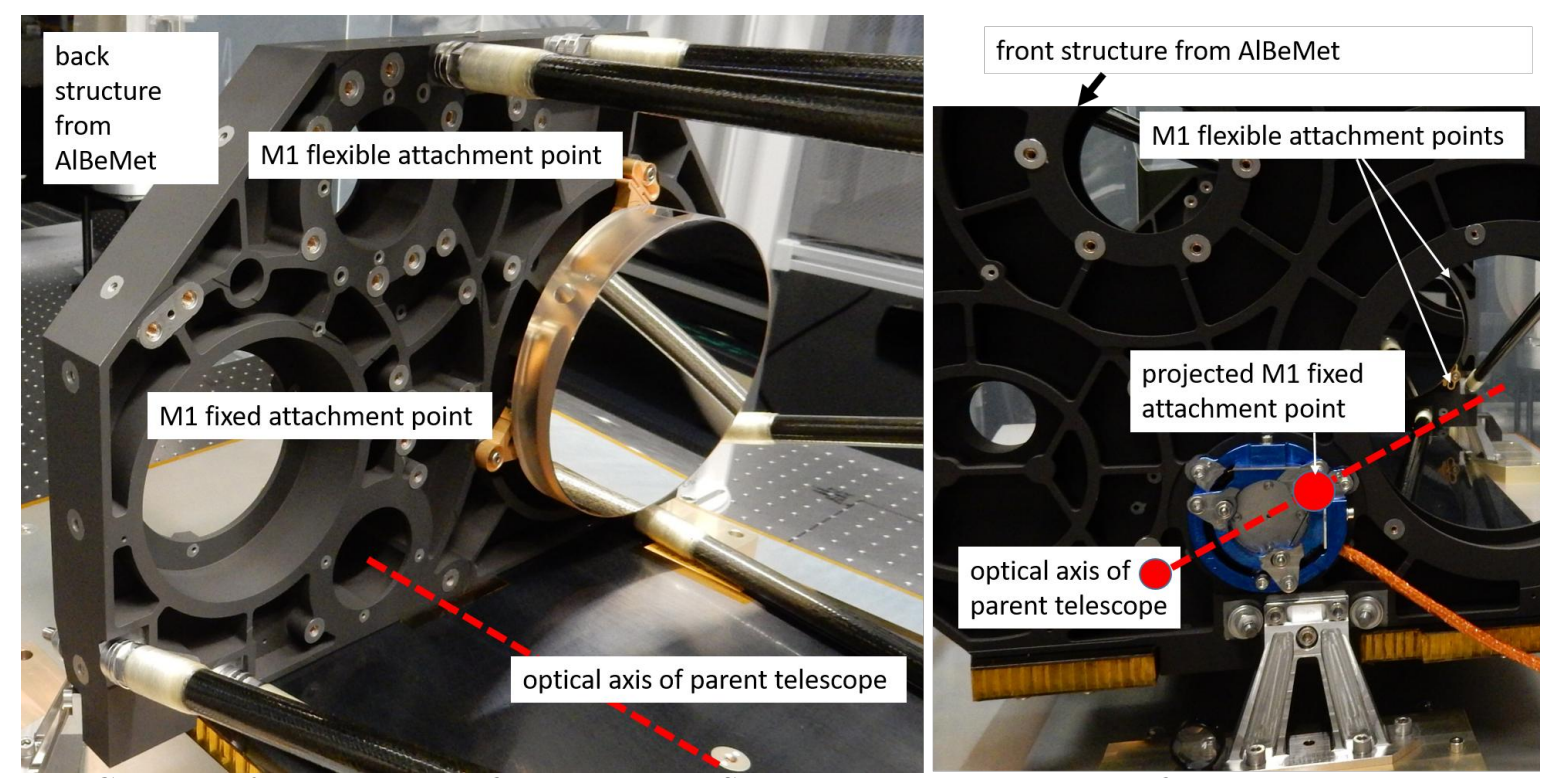

Figure 3: Concept of the decentred fixation point. Since the mirrors cannot be fixed on the optical axis of the parent telescope, we chose a common fixed point between the M1 and M2 centers. This ensures that the lateral mirror separation is within tolerances even under thermal expansion of the AlBeMet blocks. See text for details.

wide operational temperature range of PHI, this poses high demands on the thermal expansion of the mirrors, their cells, and the main structure, into which both mirrors are mounted. When considering that the HRT is a decentred system with a decentration of $170 \mathrm{~mm}$ in the aperture plane, then it is evident that the physical mirror centers are far from the optical axis of the rotationally symmetric parent telescope. In our case M1 is offset by $170 \mathrm{~mm}, \mathrm{M} 2$ is off by $44 \mathrm{~mm}$ from the optical parent axis.

In a truly athermal system, these values should be kept constant for all operational temperatures. If we - however - accept a slight shift in the focal plane (which we can safely do thanks to the large corrected field-of-view of the Ritchey-Chrétien telescope), then it is sufficient to keep the distance between M1 and M2 $(170 \mathrm{~mm}-44 \mathrm{~mm})$ within its optical tolerance. Even this would require a structure material with vanishing coefficient of thermal expansion (CTE). The problem is tackled through a trifold approach: 1) mirror mounting concept, 2) material selection of the mirror cells, 3) material selection of the main structure end blocks. The basic idea behind the mounting concept of the two mirrors is to define a fixed point for each of the two mirrors with a minimum lateral separation in between them. With this approach the problem can be split into two remaining issues: the intrinsic expansion of the mirror assemblies under temperature and a potential differential expansion between the front and the rear blocks due to temperature gradients. The contribution of the mirror assemblies itself has been mitigated through selecting ultra low expansion materials such as Zerodur and Invar for the mirror assemblies (see section 3.2). A selection of similar materials to cope with the differential expansion between the blocks is not realistic due to mass restrictions (Invar) or due to the high accommodation needs (in case of carbon fibre reinforced plastic CFRP). Finally AlBeMet 162, an aluminum beryllium alloy, has been found to be the optimum compromise between low thermal expansion coefficient, thermal conductivity, density, stiffness, and machinability.

\subsection{Mirror cell design}

The PHI mirror cells were designed to be operational within the temperature range of $-30^{\circ} \mathrm{C}$ to $+90^{\circ} \mathrm{C}$ and to withstand a quasi-static load of $70 \mathrm{~g}$. A robust mechanical design was developed to meet these needs and over all to guarantee a supreme optical performance under the extreme conditions in orbit. Due to the combination of extreme temperatures and strong loads, a bipod clamping was preferred over a glue-based solution to secure the mirrors to the structure. 
a)

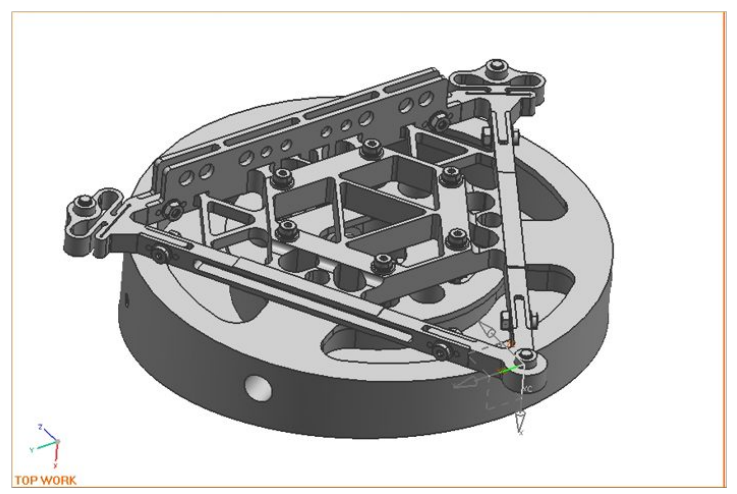

b)

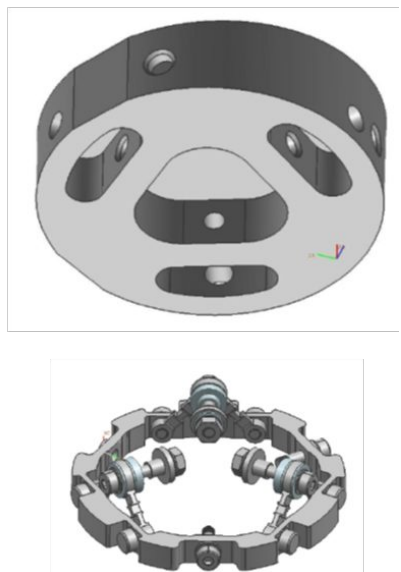

Figure 4: a) Sketch of the M1 mirror cell, with the fixed point (lower right) and the two flex blade attachment points (left and upper right). b) Sketch of the M2 mirror cell. The lightweighted Zerodur substrate (top) is mounted by three INVAR bipods. An INVAR ring (bottom) serves as buffer between the INVAR bipods and the titanium holder (not shown), onto which the M2 mirror cell is mounted. Adapted from Bischoff et al. (2014).

\subsubsection{M1 mirror cell}

The cell of the lightweighted ZERODUR M1 (shown in figure 4a) consists of a triangular mount, which uses a series of flexures to maintain the fixed point of the mirror. The fixed point for this design is the mounting hole seen at the bottom of figure $4 \mathrm{a}$. Because the mount is made of hardened Invar and Invar 36, and the interface is made of an aluminum-beryllium alloy (AlBeMet 162), there will be a substantial displacement at the two non-fixed mounting points. A double plate flexure is used at these locations to reduce the stress in the mount. The beam connecting the non-fixed mounting points is stiffened by a bar on the bottom side. This is used to move the hotspot of the first pump eigenmode to the center of the bipod assembly (for details see Bischoff et al., 2014).

\subsubsection{M2 mirror cell}

The M2 mirror acts as the tip-tilt mirror for internal image motion compensation. It is therefore mounted onto a piezo-actuator, which gets control signals from the Image Stabilisation System. The mechanical connection between the mirror and the piezo actuator (which is made from Titanium) is done by an Invar adapter, which is used as a buffer between the bipods and the titanium. The bipods, which hold the leightweighted ZERODUR mirror (see upper part of figure $4 \mathrm{~b}$ ), are secured to the mirror by Invar pins. See lower part of figure 4b. More information on the M2 mirror cell can be found in Bischoff et al. (2014).

\subsection{Protection from intense solar load, false-light and ghost suppression}

Both telescopes are protected from the intense solar flux by special heat-rejecting entrance windows, which are part of the heat-shield assembly of the spacecraft. These multilayer filters have more than $80 \%$ transmittance in a 30nm wide transmission band around the science wavelength, while effectively blocking the remaining parts of the spectrum from $200 \mathrm{~nm}$ to the far infrared by reflection. Only a small fraction of the total energy is absorbed in the window, which acts as a passive thermal element by emitting part of the thermal radiation to cold space. Emission of infrared radiation into the instrument cavity is minimized by a low emissivity coating on the backside of the window (acting at the same time as an anti-reflective coating for the science wavelength). Thus the heat load into the instrument can be substantially reduced, while preserving the high photometric and polarimetric accuracy of PHI.

The HRT channel only observes a fraction of the solar disk and must therefore be equipped with a system of internal field stops, both for thermal and for false-light control. 
a)

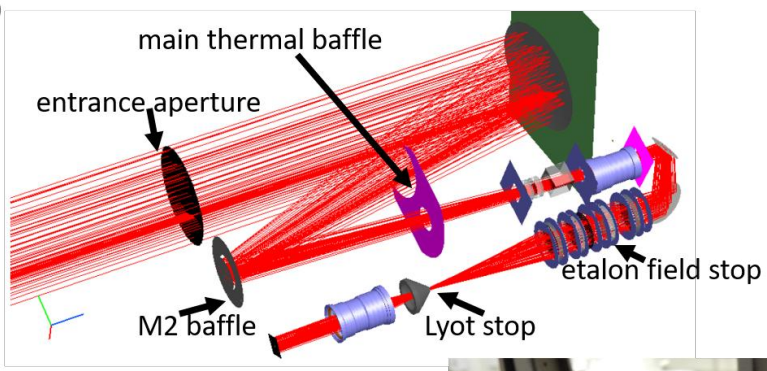

b)

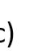

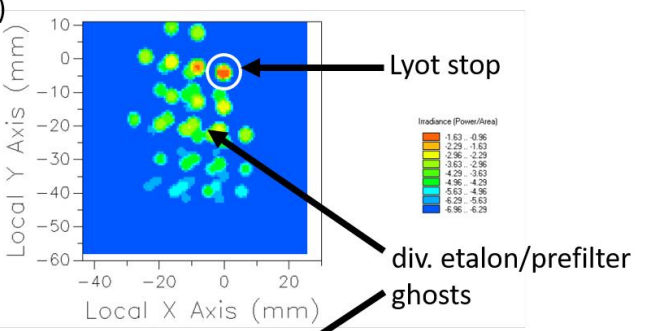

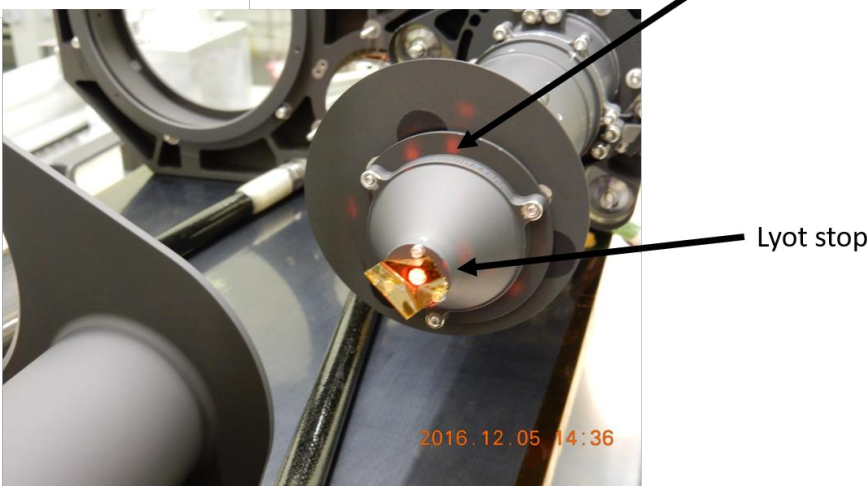

Figure 5: a) Ray trace of the science FOV showing the most important apertures and field stops of PHI HRT. b) Energy distribution in the Lyot stop plane. All etalon/prefilter ghosts are blocked by the Lyot stop. c) same during the assembly (the Lyot aperture was sealed with a Kapton tape for cleanliness).

Since we use a negative magnifier (Barlow system), neither the primary nor the secondary focus of the Ritchey-Chrétien telescope are real foci, which would allow to place a field stop. In order to block unwanted portions of the solar disk and in order to reduce the thermal load in the instrument, a series of unsharp stops is used. At each stop, a fraction of the solar beam is absorbed, without vignetting the science beam. The closer the stops are located towards the secondary focus, the sharper they become, but the less energy they get. Before the magnifying lens group, the primary role of the stops is to trap the unwanted optical energy from the solar disk outside the science FOV and thus to reduce the heat load to the optical components downstream. During the elliptical orbit of Solar Orbiter, the relative fraction and the total amount of the solar load, which is absorbed by these vanes, changes, while the fraction and the total value of the energy being passed by the vanes are constant. This is of high importance, since the polarimeter and the Filtergraph need constant working temperatures.

It is not the primary role of these vanes to act as stray-light vanes; this part is taken over by the baffling system in the common optical path. The first accessible image plane is the etalon focus, which is equipped with a physical field stop. The transfer path between the etalon and the detector is designed to contain a real image of the pupil of the system. In this pupil, a Lyot type stop is placed, which is the most efficient and most important part of the false-light suppression system of PHI.

The common path baffle provides a light tight seal between the white-light compartment before the filtergraph and the monochromatic part of the optical train behind the etalon (see Fig. 6a). It consists of a tube with internal vanes, which connects the FG housing with the camera lens tube carrying the Lyot stop.

\section{HRT MIRROR ALIGNMENT}

The alignment of a decentred Ritchey-Chrétien telescope is a challenging task. Both mirrors are free-form aspheres and must therefore be correctly aligned not only in all three translatory degrees-of-freedom (DoF), but also in all three rotatory degrees-of-freedom. The situation is further aggravated by the fact that the hyperbolic primary mirror does not produce a corrected image, not even on the optical axis. Thus the primary focus cannot be used to determine the optical axis of the system during the alignment process. To overcome these difficulties it is indispensable to take the telescope alignment already into account during the mirror specification 
a) common path baffle (upper enclosure removed)

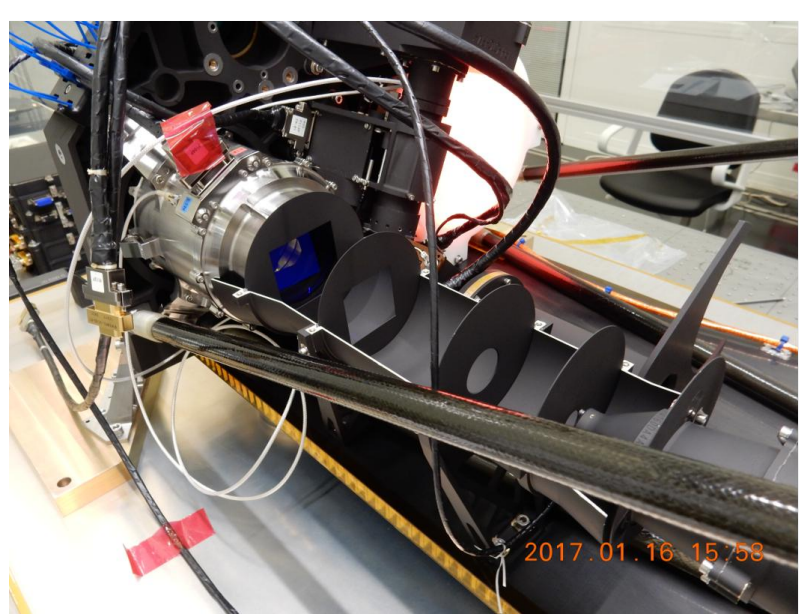

b) M2 collar and main thermal baffle as seen from M1

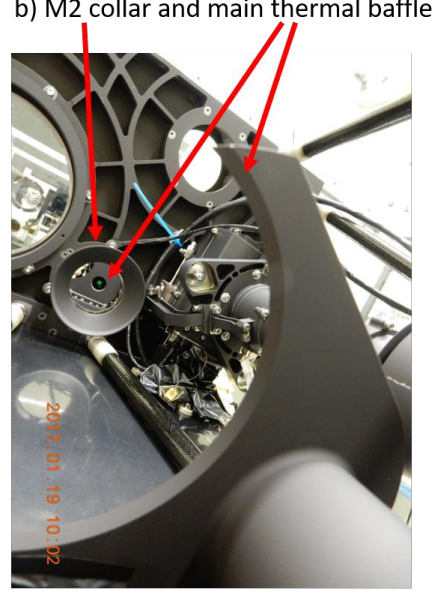

Figure 6: a) View of the common path baffle during assembly. The upper part of the tube is not yet mounted. b) View on the M2 mirror from M1. The M2 collar and the main thermal baffle can be seen. In the mirror, the main thermal baffle is seen with the PMP entrance hole. The science field can be recognized as the green light in the center shining through.

and manufacturing. Alignment marks and alignment mirror flats must be foreseen on both mirrors and must be carefully referenced to the optical surface during the manufacturing and verification process of each individual mirror. The design and fabrication of both mirror cells was managed by Carl Zeiss Optronics (later named Cassidean Optronics, then Airbus Defence and Space Optronics), the leightweighting, grinding, and polishing was done by Carl Zeiss Jena. The specifications and the manufacturing of the M1 and M2 mirror cells have been described by Bischoff et al. (2014).

In the PHI instrument the alignment of M1 with respect to the structure is possible in tip/tilt and piston (focus) only. Therefore, the exact positioning of the mirror in its cell, as well as the correct determination of the optical axis of the mirror cell is important. M2 can be aligned in the main structure with respect to M1 in all 6 degrees of freedom. In order to control the orientation of the mirror cells, both mirror substrates carry two alignment flats each, which are polished during the manufacturing of the mirror and which are referenced optically to the mirror front surface. M1 has in addition a polished back side, which defines the optical axis of the mirror during the manufacturing. This backside determines the nominal line-of-sight of the HRT telescope and must be visible during the alignment of the HRT. To this end, the PHI structure has a view-hole that allows the mirror to be viewed by a theodolite in autocollimation mode. The rotation of the M1 mirror substrate is measured by an alignment flat on the side of the mirror. It can be referenced, but the primary mirror cell cannot be shimmed in rotation within the PHI structure. The pitch angle of the M1 cell can be referenced to the vertical direction by an alignment flat on top of the M1 substrate. Since this surface can be seen through a theodolite only via a $45^{\circ}$ auxiliary folding mirror, the internal gravity reference of the theodolite looses its meaning, and another reference must be used. We used a mirror surface of liquid Galinstan (a liquid alloy of Gallium, Arsenic, and Indium, which is used in thermometers). This liquid mirror is placed under the primary mirror cell during the alignment procedure and serves as an always perfectly horizontal reference surface, which can be viewed simultanously with the M1 top alignment flat (see Fig. 7). The M2 mirror cell does not allow for a viewing of the polished back-side, such that one has to rely purely on the two alignment flats on the side and the top of the mirror substrate, respectively. With these alignment flats it is in principle possible to orient the primary and the secondary mirrors in space until they are parallel to each other. When this is reached, the mirrors must still be aligned in the three translatory degrees of freedom in order to form a corrected system. After initial mounting of the primary mirror cell and referencing of the alignment flats, the secondary mirror cell is mounted into the PHI structure. Shimming allows the mirror to be positioned and oriented in space until the alignment flats of M2 are properly oriented with respect to the ones of M1. From this point in time, the two mirror telescope will have a relatively small error in tip and tilt of M2, but still a large uncertainty in the M2 position. The telescope 


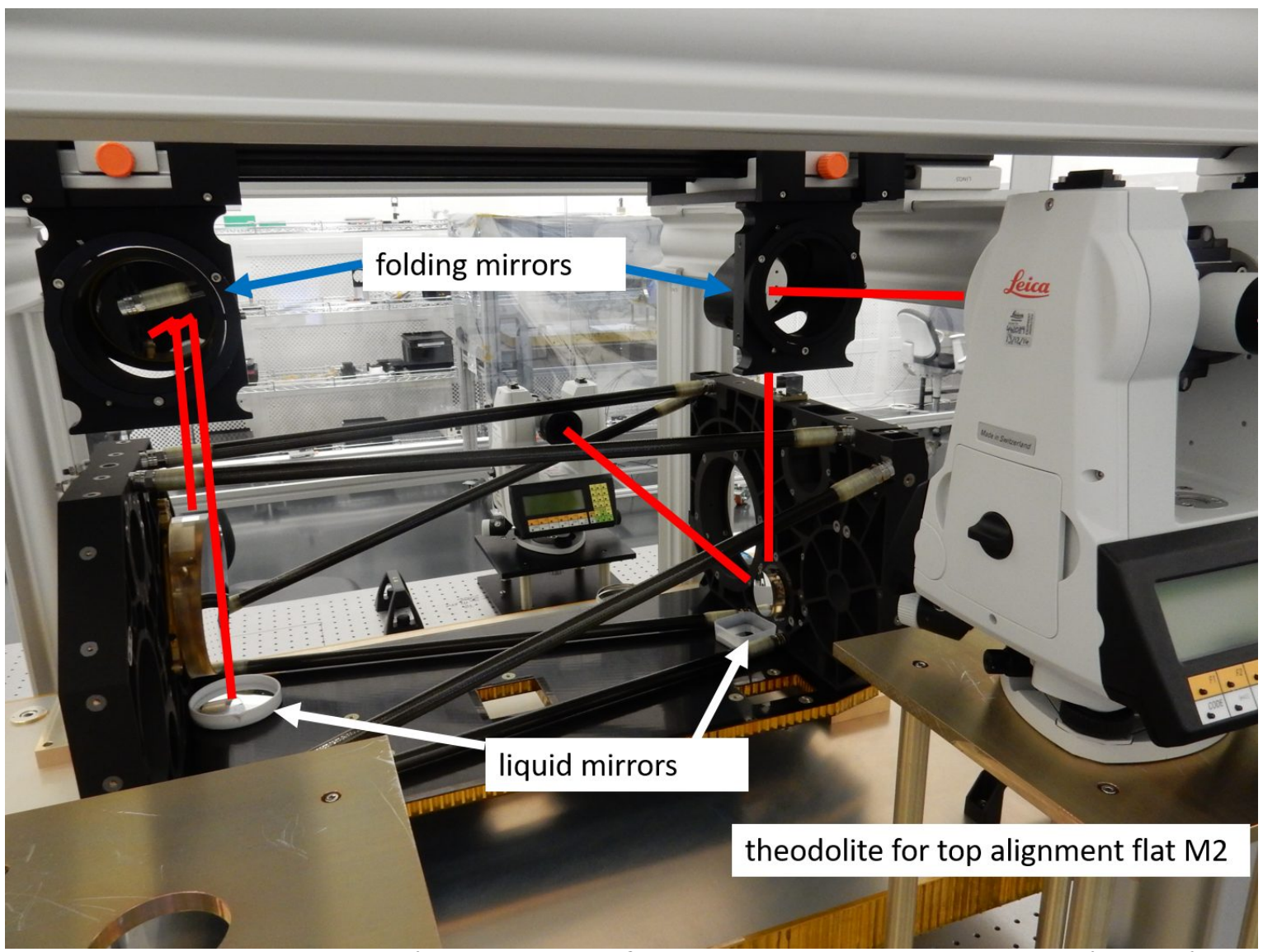

Figure 7: Theodolite measurement of the alignment flats. Liquid mirrors provide the reference for the top alignment flats, which are viewed via auxiliary folding mirrors. The side alignment flats are measured from the other side. Only the M2 theodolites are shown here, M1 measurement is done analogously.

will show large contributions of astigmatism, focus, and coma.

\subsection{Fine alignment using interferometer}

When this state is reached, we start an iterative fine alignment with the help of an interferometer in double-pass configuration. To this end, we fix the secondary focus of the telescope to its nominal position. This is the only possible choice, since otherwise the system has too many unknowns. The secondary focus is marked by a cross-hair target, which is attached to the PHI structure with close mechanical tolerances. A ZYGO 4-inch interferometer, equipped with an F/7.3 reference, provides a real interferometer focus. The interferometer is placed behind the PHI optics unit and the focus is aligned with high precision to the cross-hair target. The light from the interferometer passes the two mirror system and is reflected back by a flat high-precision reference mirror in front of the PHI HRT aperture. This reference has been initially aligned such that its surface is parallel to the polished backside of M1, which we use as the reference for the line-of-sight of the telescope. Since the system is now over-determined (line-of-sight and focus position!) it will be (due to the residual misalignment and the tolerances in the system) impossible to obtain a perfect interferogram by just shimming M2. As an additional compensator, the line-of-sight (tip/tilt of reference mirror) will be used, since the necessary corrections (and associated line-of-sight errors) are already small enough to be acceptable. The first interferogram will show astigmatism and coma, as well as defocus. With this information it is possible to feed a mathematical optical model and to determine - by a least square fit of the aberrations to the model - the combination of decenter, piston, and tip/tilt of M2. Depending on the quality of the initial alignment, typically three to four iterations are needed until a perfect wave-front performance is reached (then the shim step proposed by the computer falls below $0.01 \mathrm{~mm}$, the thinnest shims we used). 


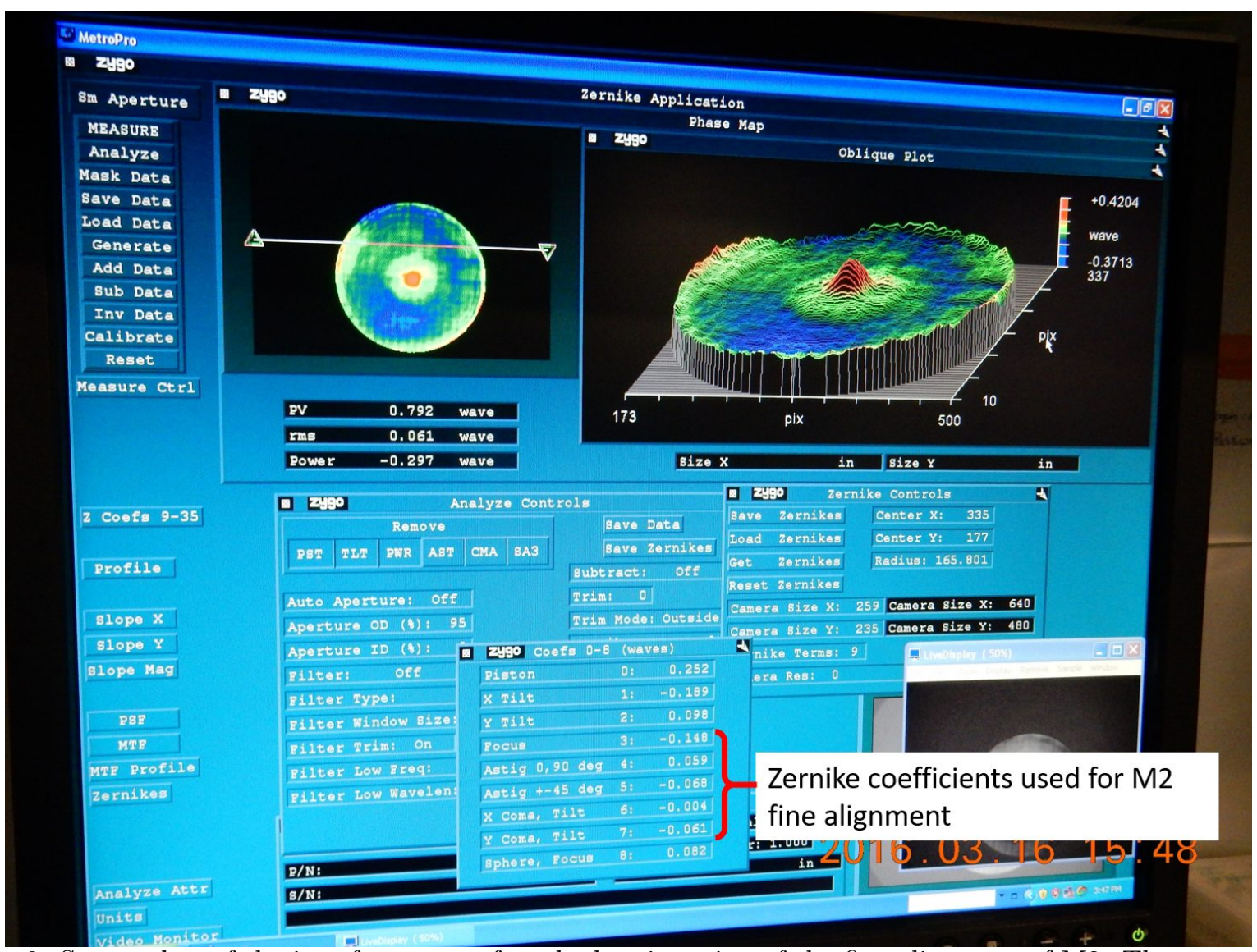

Figure 8: Screen shot of the interferometer after the last iteration of the fine alignment of M2. The output of a Zernike fit to the interferogram is used as the input for a mathematical optical model.

\section{VERIFICATION APPROACH}

The optical performance of the HRT channel is measured in different stages of the alignment by different means. The mirror system M1 and M2, and the magnified telescope consisting of M1, M2, and the magnifier lens group, are measured with an interferometer in double pass, both, on axis and in distinct positions spread over the entire field-of-view.

Once the filtergraph is mounted, an interferometric measurement is no longer possible, since the system is built to transmit the science wavelength of $617.3 \mathrm{~nm}$ only, and thus will be opaque to the LASER light. We have to this end produced an identical filtergraph for alignment and verification of the common path. This alignment filtergraph is optically identical to the flight version, with the exception that it contains no etalon. For the optical verification this is, however, tolerable, since the etalon is placed in an intermediate focal plane of the common path, and the optical properties of the etalon are almost invisible in the systems WFE performance. The assessment of the optical performance (image geometry and quality) of the fully assembled system with the flight filtergraph and the science focal plane array is done by stimulating the HRT channel with a "counter-telescope" or "stimulus-telescope". This stimulus telescope is based on a telescope lens of the AS type produced by Carl Zeiss Jena. We used an AS 150/2250 air spaced achromatic lens with a measured WFE of lambda/91 at $632 \mathrm{~nm}$. This telescope can be equipped with a set of optical targets for the determination of geometric image properties and image quality.

Targets include a Siemens star, grids, cross-hairs, point arrays (negative and positive), pinhole, USAF target, and a random grey pattern. The telescope is illuminated by a high power LED from Thorlabs with an electric power of up to $3 \mathrm{~W}$ with a spectral output containing the $617.3 \mathrm{~nm}$ science wavelength of PHI. For optical 


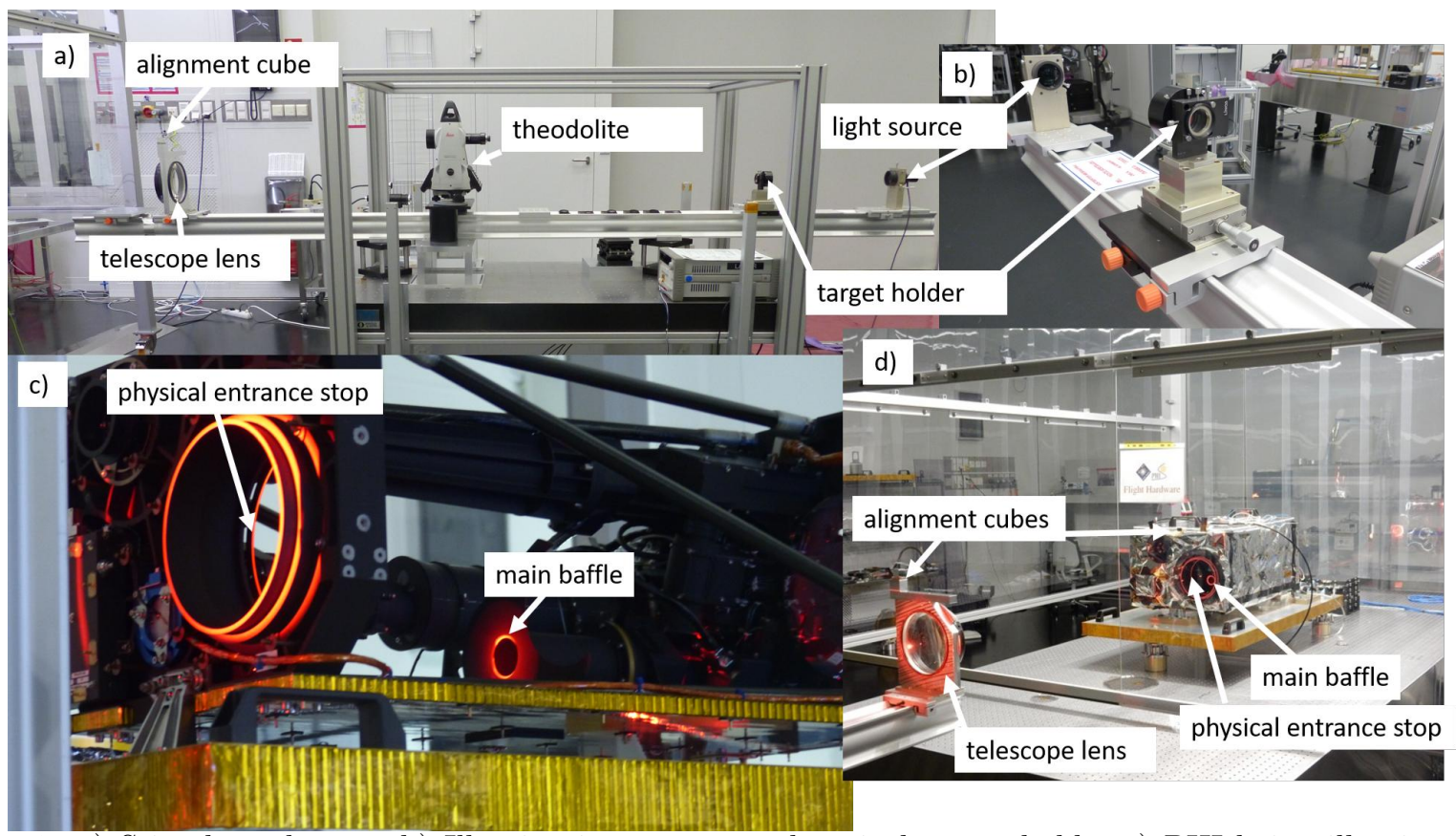

Figure 9: a) Stimulus telescope b) Illumination system and optical target holder c) PHI being illuminated by the stimulus telescope during assembly d) PHI being illuminated during the outgoing test prior to delivery. The red illuminated HRT aperture and the PMP stop (main baffle) can clearly be recognised.

stimulation of the HRT with real sunlight, a $53 \mathrm{~cm}$ aperture 2-mirror coelostat at MPS premises was used. The solar light captured by the coelostat was used to feed PHI with sunlight for the spectral calibration, while the instrument was in the vacuum chamber. A description of this test can be found in Fernández-Rico et al. (2018).

\section{ON GROUND OPTICAL PERFORMANCE}

Optical tests and calibrations on ground confirmed the high optical quality of the HRT channel. The system is diffraction limited and all optical parameters are within tolerances. Furthermore, this high performance can be kept over the large observational temperature range. A detailed quantitative discussion of the calibration data goes beyond the scope of this paper and will be presented in a dedicated later publication. The ghost suppression works perfectly, and we could not identify disturbing interference fringes in the focal plane. The polarimetric behaviour of the telescope is nominal, as is the spectral transmission, which was tested in vacuum conditions with real sunlight.

\section{CONCLUSIONS}

The HRT channel of the PHI instrument showed perfect optical performance in laboratory conditions, as well as during the thermal vacuum tests. The system in-flight performance is not expected to be limited by the alignment between M1 and M2; the HRT telescope has been designed and tested for a temperature range, which is much larger than the latest predictions for the orbital temperature variations, which have been calculated by the spacecraft provider Airbus Defence and Space UK. The concept of the decentred fixation points of the mirror cells worked perfectly. The HRT channel preserves the high polarimetric efficiency and the very high spectral resolution of the filtergraph. 


\section{Acknowledgements}

The authors would like to express their gratitude to the team of Carl Zeiss Optronics for the very fruitful collaboration in the development of the mirror cells for PHI. Solar Orbiter is a mission led by the European Space Agency (ESA) with signicant contribution from National Aeronautics and Space Administration (NASA). The SO/PHI instrument is supported by the German Aerospace Center (DLR) through grants 50 OT 1201 and 50 OT 1703 (for the ISS) and a grant from the Max Planck Society for Solar Orbiter instrument development. The Spanish contribution has been partly funded by the Spanish Research Agency under project ESP2016-77548-C5 and ESP2014-56169-C6, partially including European FEDER funds.

\section{REFERENCES}

[1] Álvarez-Herrero, A., García Parejo, P., Laguna, H., Villanueva, J., Barandiarán, J., Bastide, L., Reina, M., Sánchez, A., Gonzalo, A., Navarro, R., Vera, I., and Royo, M., "Polarization modulators based on liquid crystal variable retarders for the Solar Orbiter mission," in [Polarization Science and Remote Sensing VII], Society of Photo-Optical Instrumentation Engineers (SPIE) Conference Series 9613, 96130I (Sept. 2015).

[2] Barthol, P., Gandorfer, A., Solanki, S. K., Schüssler, M., Chares, B., Curdt, W., Deutsch, W., Feller, A., Germerott, D., Grauf, B., Heerlein, K., Hirzberger, J., Kolleck, M., Meller, R., Müller, R., Riethmüller, T. L., Tomasch, G., Knölker, M., Lites, B. W., Card, G., Elmore, D., Fox, J., Lecinski, A., Nelson, P., Summers, R., Watt, A., Martínez Pillet, V., Bonet, J. A., Schmidt, W., Berkefeld, T., Title, A. M., Domingo, V., Gasent Blesa, J. L., Del Toro Iniesta, J. C., López Jiménez, A., Álvarez-Herrero, A., Sabau-Graziati, L., Widani, C., Haberler, P., Härtel, K., Kampf, D., Levin, T., Pérez Grande, I., Sanz-Andrés, A., and Schmidt, E., "The Sunrise Mission," Solar Physics 268, 1-34 (Jan. 2011).

[3] Bischoff, J., Grauf, B., Staub, J., Gandorfer, A., Woch, J., Clark, S., Zimmermann, M., Kolb, A., Metz, B., and Rucks, P., "Development of mirror cells for a satellite born solar telescope," in [Shaping the Future by Engineering], proceedings Ilmenau Scientific Colloquium 58 (Sept. 2014).

[4] Carmona, M., Gómez, J. M., Roma, D., Casas, A., López, M., Bosch, J., Herms, A., Sabater, J., Volkmer, R., Heidecke, F., Maue, T., Nakai, E., and Schmidt, W., "System model of an image stabilization system," in [Modeling, Systems Engineering, and Project Management for Astronomy VI], 9150, 91501U (Aug. 2014).

[5] Fernández-Rico, G., Álvarez-Copano, M., Deutsch, W., Gandorfer, A., Ramanath, S., Staub, J., Bambach, P., and Torralbo, I., "Thermal Vacuum and Balance Test of the ESA Solar Orbiter Instrument PHI," International Conference on Environmental Systems 2018.

[6] Gandorfer, A., Solanki, S. K., Woch, J., Pillet, V. M., Álvarez Herrero, A., and Appourchaux, T., "The solar orbiter mission and its polarimetric and helioseismic imager (SO/PHI)," Journal of Physics: Conference Series 271(1), 012086 (2011).

[7] Gensemer, S. D. and Farrant, D., "Fabrication and metrology of lithium niobate narrowband optical filters for the solar orbiter," Advanced Optical Technologies 3, 309-314 (June 2014).

[8] Marsden, R. G. and Müller, D., [Solar Orbiter: Linking the Sun and Inner Heliosphere], 347 (2011).

[9] Martínez Pillet, V., Del Toro Iniesta, J. C., Álvarez-Herrero, A., Domingo, V., Bonet, J. A., González Fernández, L., López Jiménez, A., Pastor, C., Gasent Blesa, J. L., Mellado, P., Piqueras, J., Aparicio, B., Balaguer, M., Ballesteros, E., Belenguer, T., Bellot Rubio, L. R., Berkefeld, T., Collados, M., Deutsch, W., Feller, A., Girela, F., Grauf, B., Heredero, R. L., Herranz, M., Jerónimo, J. M., Laguna, H., Meller, R., Menéndez, M., Morales, R., Orozco Suárez, D., Ramos, G., Reina, M., Ramos, J. L., Rodríguez, P., Sánchez, A., Uribe-Patarroyo, N., Barthol, P., Gandorfer, A., Knoelker, M., Schmidt, W., Solanki, S. K., and Vargas Domínguez, S., "The Imaging Magnetograph eXperiment (IMaX) for the Sunrise Balloon-Borne Solar Observatory," Solar Physics 268, 57-102 (Jan. 2011).

[10] Mueller, D., Marsden, R. G., Cyr, O. S., Gilbert, H. R., et al., "Solar orbiter," Solar Physics 285(1-2), 25-70 (2013).

[11] Solanki, S. K., Barthol, P., Danilovic, S., Feller, A., Gandorfer, A., Hirzberger, J., Riethmüller, T. L., Schüssler, M., Bonet, J. A., Martínez Pillet, V., del Toro Iniesta, J. C., Domingo, V., Palacios, J., Knölker, M., Bello González, N., Berkefeld, T., Franz, M., Schmidt, W., and Title, A. M., "SUNRISE: Instrument, Mission, Data, and First Results," The Astrophysical Journal Letters 723, L127-L133 (Nov. 2010). 
[12] Solanki, S. K., del Toro Iniesta, J. C., Woch, J., Gandorfer, A., Hirzberger, J., Schmidt, W., Appourchaux, T., and Alvarez-Herrero, A., "The Polarimetric and Helioseismic Imager for Solar Orbiter: SO/PHI," in [Polarimetry: From the Sun to Stars and Stellar Environments], Nagendra, K. N., Bagnulo, S., Centeno, R., and Jesús Martínez González, M., eds., IAU Symposium 305, 108-113 (Oct. 2015).

[13] Volkmer, R., Bosch, J., Feger, B., Gomez, J. M., Heidecke, F., Schmidt, W., Scheiffelen, T., Sigwarth, M., and Soltau, D., "Image stabilisation system of the photospheric and helioseismic imager," in [Space Telescopes and Instrumentation 2012: Optical, Infrared, and Millimeter Wave], Society of Photo-Optical Instrumentation Engineers (SPIE) Conference Series 8442, 84424P (Sept. 2012). 